\title{
Detection of contaminant plumes released from landfills: numerical versus analytical solutions
}

\author{
N. Buket Yenigül · André T. Hensbergen • \\ Amro M. M. Elfeki • F. Michel Dekking
}

Received: 17 July 2009/Accepted: 17 March 2011/Published online: 16 April 2011

(C) The Author(s) 2011. This article is published with open access at Springerlink.com

\begin{abstract}
Contaminant leaks released from landfills are a threat to groundwater quality. The groundwater monitoring systems installed in the vicinity of such facilities are vital. In this study the detection probability of a contaminant plume released from a landfill has been investigated by means of both a simulation and an analytical model for both homogeneous and heterogeneous aquifer conditions. Since the detection probability is a sensitive quantity, we first compare the two methods for homogeneous aquifer conditions to assess the errors that are encountered by performing simulations. The analysis shows that the simulation model yields the detection probabilities of a contaminant plume at a given monitoring well quite well in the homogeneous case. For heterogeneous aquifers we apply the approximated analytical model based on macro-dispersivities. Here we find that this model is insufficient in monitoring system design, since the obtained analytical values of the detection
\end{abstract}

A. M. M. Elfeki is on leave from Irrigation and Hydraulics Department, Faculty of Engineering, Mansoura University, Mansoura, Egypt.

\section{N. B. Yenigül}

Faculty of Civil Engineering and Geosciences,

Water Resources Section,

Delft University of Technology, Delft, The Netherlands

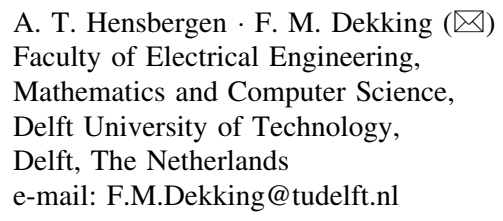

\section{A. M. M. Elfeki}

Faculty of Meteorology, Environment and Arid Land Agriculture, Department of Hydrology and Water Resources Management, King Abdulaziz University, Jeddah, Saudi Arabia probabilities at a given monitoring well differ significantly from those computed by simulation.

Keywords Contamination · Plumes · Landfills · Detection probability $\cdot$ Monitoring wells

\section{Introduction}

Contaminants are introduced into the groundwater by human activities rather than natural ones. Landfills, storage, and transportation of commercial materials, mining, agricultural operations, interaquifer exchange, and saltwater intrusion are the major sources of groundwater contamination. Among these, landfills represent a widespread and significant threat to groundwater quality, human health, and even more to some of the ecosystems. In communal language, landfill means waste disposal on land. However, technically one may define landfill as "the engineered deposit of waste onto or into land in such a way that pollution or harm to the environment is prevented and through restoration of land provided which may be used for other purpose" (Bagchi 1994). Unfortunately, despite an ideal site selection and a thriving design, on several occasions the environmental impact of landfill leakage, particularly on groundwater quality, has been quite severe. Works by Mikac et al. (1998), Tatsi and Zouboulis (2002), Chofqi et al. (2004), are only a few of the countless examples presented in the literature. The risk of groundwater contamination can be further reduced by installing a monitoring system composed of a series of wells located around the landfill and sampled periodically for contaminants. However, it is difficult to ensure that a specific monitoring system will detect all of the contaminants released from the landfill because of the numerous and 
significant uncertainties involved. Size and location of the possible contaminant leak, spatial variability of the hydrogeological characteristics (which make groundwater flow and contaminant paths hard to predict), locations, depths and number of monitoring wells, chemical characteristics of contaminants and sampling procedures are the sources of uncertainties that have a great influence on the efficiency of a monitoring system.

Several studies on the monitoring problem have been presented in the literature. Most of these studies do not incorporate all the relevant factors due to the complexity of the issue. In general approaches based on geostatistical methods (i.e., Rouhani and Hall 1988; Haug et al. 1989), optimization methods (i.e., Hudak and Loaiciga 1993; Meyer et al. 1994; Storck et al. 1997), methods based on extensive simulation (Massmann and Freeze 1987; Meyer et al. 1994; Storck et al. 1997) and graphical methods (Hudak 2001, 2002) are used to design monitoring systems.

In this study, the detection probability of a plume released from a landfill has been investigated by means of both a simulation and an analytical model. Analytical models are generally available only for very simplified situations such as a homogeneous medium and a uniform flow field. Simulations are used in case of heterogeneous media. The assumption of homogeneous field conditions in groundwater flow problems may yield an appropriate approximation in some situations. In contamination problems, however, the extent and characteristics of a contaminant plume may be significantly influenced by the heterogeneous nature of geologic formations. Areas of low conductivity may slow the flow and reduce the spreading of a plume, whereas high conductivity zones may cause channeling of the plume. These types of regimes cannot be properly analyzed under assumptions of a homogeneous medium. Still, the significance of analytical models should not be underestimated, as they are important tools to verify the simulations and to obtain a thorough understanding of the phenomena. The detection probability is a particularly sensitive quantity; therefore, we compare its analytical determination with the results from simulations first for homogeneous aquifers. Thus, we obtain an idea of the errors that might be encountered with the simulation model and we have an opportunity to study the sensitivity of the parameters.

In the second part of the paper, we follow the same scenario for heterogeneous aquifers. Since there is a general agreement that conductivity variations play an important role in contaminant transport, a very primitive assumption for homogenization of a heterogeneous medium might be to use an effective conductivity. This may result in an overestimation of the velocity and extent of the plume. Consequently, this may result in very conservative and costly monitoring. On the other hand, if a very small conductivity is used, non-conservative designs may result in underestimation of the contaminant plume. In the last two decades, a significant amount of research has been devoted to understand the effects of natural heterogeneity on solute transport and to the development of modeling techniques which explicitly account for natural heterogeneity (e.g. Gelhar et al. 1979; Gelhar and Axness 1983; Dagan 1984, 1986 Rubin 1990; Thompson and Gelhar 1990; Vomvoris and Gelhar 1990; Kapoor and Gelhar 1994a, b; McLaughlin and Ruan 2001; Hu et al. 2002). Apparently, modeling of contaminant transport using an advection-dispersion equation with macro-dispersivities is a common practice. The macro-dispersion coefficient embodies the effect of unresolved advective heterogeneity on the spatial second moment and can be used to describe the average concentration distribution. In this study, the mean concentration field is determined using the macro-dispersion coefficient in the analytical model (e.g., Kapoor and Gelhar 1994a, b). Here the macro-dispersion coefficient is the summation of the local dispersivities and constant macro-dispersivities as computed by Gelhar and Axness (1983) and the detection probability of the contaminant plume is computed for a homogenized aquifer (it is assumed that the plume traveled enough correlation scales to reach the asymptotic macrodispersivities, which is achieved in the model because the wells are far enough from the landfill to reach the asymptotic regime). The results of the analysis based on the simulation and analytical models are compared to find the answers to the following questions: How far an analytical model can be used in groundwater monitoring system design while incorporating the effects of various heterogeneities on contaminant transport? How accurately can the detection probability of a contaminant plume by a given monitoring well be computed by an analytical model, which uses macro-dispersivities to homogenize the heterogeneity? How large will be the discrepancies between the results obtained by the two models (simulation versus analytical)?

\section{The simulation model}

A Monte Carlo approach coupled with a two-dimensional finite difference flow model and a random walk particletracking model (adapted from Elfeki 1996) is used to simulate a large number of contaminant plumes released from a landfill. The heterogeneity of the subsurface and the leak locations are the uncertainties incorporated in the simulation model.

Two-dimensional groundwater flow model

A two-dimensional steady saturated groundwater flow in an isotropic heterogeneous aquifer in a rectangular domain of dimension $\left(0 \leq x \leq L_{x}, 0 \leq y \leq L_{y}\right)$ is given by 
$\frac{\partial}{\partial x}\left(K \frac{\partial h}{\partial x}\right)+\frac{\partial}{\partial y}\left(K \frac{\partial h}{\partial y}\right)=0$

where $K$ is the hydraulic conductivity $[L / T]$ and $h$ is the hydraulic head $[L]$. A block-centered five-point finite difference method is used to solve Eq. 1 under different boundary conditions and the inter-nodal Darcy's velocity and groundwater velocity components are computed (Elfeki 1996).

Particle-tracking random walk for contaminant transport

The movement of contaminants in the subsurface is represented by the advection-dispersion equation. The contaminant is assumed to be conservative. The twodimensional equation in this case is (Bear 1972):

$$
\begin{gathered}
\frac{\partial C}{\partial t}+v_{x} \frac{\partial C}{\partial x}+v_{y} \frac{\partial C}{\partial y}-\frac{\partial}{\partial x}\left[D_{x x} \frac{\partial C}{\partial x}+D_{x y} \frac{\partial C}{\partial y}\right] \\
-\frac{\partial}{\partial y}\left[D_{y x} \frac{\partial C}{\partial x}+D_{y y} \frac{\partial C}{\partial y}\right]=0,
\end{gathered}
$$

where $C$ is the concentration of the contaminant at time $t$ at location $(x, y)\left[M / L^{3}\right], v_{x}$ and $v_{y}$ are the average groundwater flow velocity components in the $x$ - and $y$-direction respectively, and $D_{x x}, \ldots, D_{y y}$ are the components of the pore scale hydrodynamic dispersion tensor $\left[L^{2} / T\right]$. The terms $D_{x y}$ and $D_{y x}$ only play a role if the medium is heterogeneous; in the homogeneous case they are both equal to 0. Having obtained the velocity field from the flow equation, the solution of the transport equation can be obtained under given initial and boundary conditions. In this study, the random walk particle-tracking model is used since it does not exhibit numerical dispersion (Kinzelbach 1986).

\section{Probability of detection}

A Monte Carlo simulation procedure is used to compute the detection probability, $P_{d(\mathrm{mw})}$ of a given monitoring well $(\mathrm{mw})$. First, a realization of a random hydraulic conductivity field is generated. After solving the flow model, a random leak location is generated. Then the random walk transport model is solved to determine the concentration field until the plume reaches the compliance boundary. Finally, the model checks whether the concentration value at a given monitoring well location exceeds a detection limit to determine whether a plume is detected.

Detection of a plume by a monitoring well, is defined as the event where the concentration at the well location, $C_{\mathrm{mw}}=C\left(x_{\mathrm{mw}}, y_{\mathrm{mw}}, t\right)$ at some time $t$ is equal to or greater than a given threshold concentration $C_{\mathrm{TH}}$. Therefore the probability of detection $P_{d(\mathrm{mw})}$ of a given plume by a given monitoring well is:
$P_{d(\mathrm{mw})}=P\left(C_{\mathrm{mw}} \geq C_{\mathrm{TH}}\right.$, at some time $\left.t\right)=\frac{1}{N_{\mathrm{MC}}} \sum_{i=1}^{N_{\mathrm{MC}}} I_{d}^{(i)}$

Here $N_{\mathrm{MC}}$ is the total number of simulated plumes, and $I_{d}^{(i)}$ equals 1 if the simulated plume $i$ is detected by the given monitoring well, and equals zero otherwise.

\section{The analytical model}

Homogeneous aquifers

The concentration at position $(x, y)$ and at time $t$ due to an instantaneous release of contaminant at location $\left(x_{0}, y_{0}\right)$ is given by (Bear 1972),

$$
\begin{aligned}
C(x, y, t)= & \frac{M_{0} /(\varepsilon H)}{\sqrt{4 \pi \alpha_{L} v_{x} t} \sqrt{4 \pi \alpha_{T} v_{x} t}} \\
& \times \exp \left[-\frac{\left(x-x_{0}-v_{x} t\right)^{2}}{4 \alpha_{L} v_{x} t}-\frac{\left(y-y_{0}\right)^{2}}{4 \alpha_{T} v_{x} t}\right]
\end{aligned}
$$

From this the solution in the case of continuous release follows by integration:

$$
\begin{aligned}
C(x, y, t)= & \frac{\dot{M}_{0} /(\varepsilon H)}{4 \pi v_{x} \sqrt{\alpha_{L} \alpha_{T}}} \int_{0}^{t} \frac{1}{t-\tau} \\
& \times \exp \left[-\frac{\left(x-x_{0}-v_{x}(t-\tau)\right)^{2}}{4 \alpha_{L} v_{x}(t-\tau)}-\frac{\left(y-y_{0}\right)^{2}}{4 \alpha_{T} v_{x}(t-\tau)}\right] d \tau
\end{aligned}
$$

Here $\alpha_{L}$ is the longitudinal dispersivity $[L], \alpha_{T}$ is the transversal dispersivity $[L], v_{x}$ is the plume velocity in $x$-direction $[L / T], M_{0}$ is the injected mass $[M], \dot{M}_{0}$ is the injected mass rate $[M / T], H$ is the aquifer thickness, $\varepsilon$ is the effective porosity. Our motivation to study both the instantaneous and the continuous release of contamination is that these are in fact the two extreme cases. In real landfills the actual process will be somewhere in between these extreme cases, e.g., a plume of a specific contaminant in a landfill results from a continuous release at a varying rate during some period of time.

Note that Eqs. $4 \mathrm{a}$ and $4 \mathrm{~b}$ both give a pointwise concentration whereas in the simulation model the concentration is calculated by means of particles in a grid cell. Hence one must average the concentration over the grid cells in order to make a fair comparison. Therefore a weighted average concentration with weights corresponding to Simpson's 2nd rule is used in the analytical model. In highly dispersive media and/or far away from the source the averaging does not make much difference since the plumes are already quite spread out. However for the 
locations where the plume is very peaked the effect is noticeable. But even in the region where the averaging does not matter, Simpson approximation for the integral over a grid cell gives a small bias.

To find the concentration of a plume resulting from a continuous leak two different approaches can be taken. The first approach is to approximate such a plume by repeated small instantaneous plumes at short time intervals. In fact, taking the intermittent time intervals shorter and shorter, apart from inherent numerical instability around the origin, in this way the exact concentration will be better approximated. The second approach is to use the approximation of the concentration by the Hantush well function (Kinzelbach 1986). Calculations with Matlab showed that for wells not too far from the source, the two approximations are quite close, but further away the Hantush approximation breaks down. The Hantush function looks like an elegant closed form, but the improper integral it contains limits its numerical application. For large $x$-values, numerical breakdown occurs as in the Hantush formula a very large number is multiplied with a number close to zero.

\section{Heterogeneous aquifers}

Heterogeneity can be dealt with by defining the homogeneous equivalent properties. The advection-dispersion equation that includes the effect of the variations of velocities at the local and regional scale can be written as (Kapoor and Gelhar 1994a, b):

$\frac{\partial \bar{C}}{\partial t}+v \frac{\partial \bar{C}}{\partial x_{1}}-v\left(A_{i j}+\alpha_{i j}\right) \frac{\partial^{2} \bar{C}}{\partial x_{i} \partial x_{j}}=0$

where $\bar{C}$ is the mean concentration at a regional scale $\left[M / L^{3}\right]$, $v$ is the mean regional velocity in the $x_{1}$ direction $[L / T], A_{i j}$ and $\alpha_{i j}$ are the macro- and local dispersivities $[L]$, respectively; for the indices $i$ and $j$ the Einstein summation convention applies. The mean concentration, governed by Eq. 5 for an instantaneous release of contaminant is assumed to be Gaussian and the solution can be expressed as:

$$
\begin{aligned}
\bar{C}(x, y, t)= & \frac{M_{0} /(\varepsilon H)}{\sqrt{4 \pi\left(A_{L}+\alpha_{L}\right) v_{x} t} \sqrt{4 \pi\left(A_{T}+\alpha_{T}\right) v_{x} t}} \\
& \times \exp -\left[\frac{\left(x-x_{0}-v_{x} t\right)^{2}}{4\left(A_{L}+\alpha_{L}\right) v_{x} t}+\frac{\left(y-y_{0}\right)^{2}}{4\left(A_{T}+\alpha_{T}\right) v_{x} t}\right]
\end{aligned}
$$

Theoretically derived $A_{L}$ and $A_{T}$ values are given by (Gelhar and Axness 1983),

$A_{L}=\sigma_{Y}^{2} \lambda / \gamma^{2}$ and $A_{T}=\frac{\sigma_{Y}^{2} \alpha_{L}}{8 \gamma^{2}}\left(1+3 \frac{\alpha_{T}}{\alpha_{L}}\right)$

where $\lambda$ and $\sigma_{Y}$ are the correlation length $[L]$ and the standard deviation of the $Y=\ln K$ field. $\gamma$ is a flow factor, which for the isotropic case is $\gamma=1+\sigma_{Y}^{2} / 6$ and $\gamma=1$ if it is assumed that the local dispersivity $\alpha_{L}$ is small compared to correlation length $\lambda$. In this study, $\gamma$ is considered to be 1 since $\alpha_{L}$ is taken in the order of centimeters, while $\lambda$ is in the order of meters.

\section{Probability of detection}

Plumes start from a random location $\left(x_{0}, y_{0}\right)$ where $x_{0}$ is fixed and $y_{0}$ is between $y_{c}-L$ and $y_{c}+L$ where $2 L$ is the length of the landfill. Detection of such a plume at a well located at position $\left(x_{\mathrm{mw}}, y_{\mathrm{mw}}\right)$ occurs if the concentration at the monitoring well $C\left(x_{\mathrm{mw}}, y_{\mathrm{mw}}, t\right)$ is greater than or equal to the threshold concentration $C_{\mathrm{TH}}$ at some moment in time. By calculating the maximum concentrations on the line $x=x_{\mathrm{mw}}$ the maximum width of the plume (above a given concentration threshold) at $x_{\mathrm{mw}}$ can be found (See Appendix A).

Define the detection region $D\left(x_{0}, y_{0}, C_{\mathrm{TH}}\right)$ as the set of the points $(x, y)$ where at some moment in time a plume starting from $\left(x_{0}, y_{0}\right)$ will be detected at level $C_{\mathrm{TH}}$. Likewise let the leak region $L\left(x_{\mathrm{mw}}, y_{\mathrm{mw}}, C_{\mathrm{TH}}\right)$ be the set of points $(x, y)$ such that a plume starting from $(x, y)$ will be detected by a well at location $\left(x_{\mathrm{mw}}, y_{\mathrm{mw}}\right)$. In a homogeneous medium the shape of a plume is the same whatever its starting point and the leak region and the detection region for one and the same point $(x, y)$ are each other's image under reflection in the point $(x, y)$ (see Fig. 1). Suppose that the plume released from $\left(x_{0}, y_{0}\right)$ has width $2 l$ at distance $x_{\mathrm{mw}}$ from the source. Any leak on the line $x=x_{0}$ between $y_{\mathrm{mw}}-l$ and $y_{\mathrm{mw}}+l$ will be detected; any leaks with other $y$ values will not. The detection probability is thus simply the fraction of the line segment $x=x_{0}, y_{c}-L \leq y \leq y_{c}+L$ that is covered by $\left[y_{\mathrm{mw}}-l, y_{\mathrm{mw}}+l\right]$. As long as $l<L$ and $\left[y_{\mathrm{mw}}-l, y_{\mathrm{mw}}+\right.$ l] falls completely within $\left[y_{c}-L, y_{c}+L\right]$, which happens if
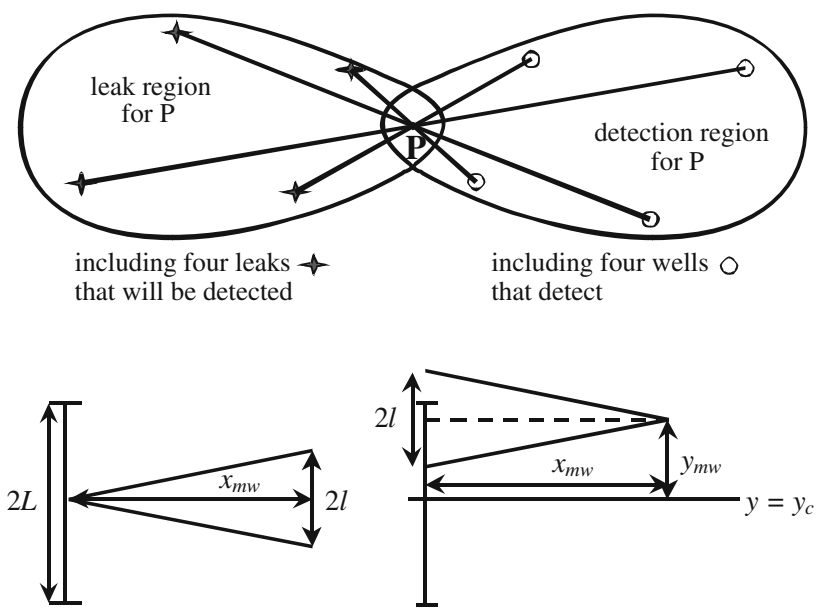

Fig. 1 Depiction of detect and leak regions 
$y_{c}-L+l \leq y_{\mathrm{mw}} \leq y_{c}+L-l$, the detection probability is therefore

$P_{d(\mathrm{mw})}=\frac{2 l}{2 L}=\frac{l}{L}$.

When calculating the detection probability of a well close to the boundaries, or in case $L \leq l \leq 2 L$, a boundary effect should be taken into account (See Appendix B). Last of all, if $l>2 L$ then any leak within $\left[y_{c}-L, y_{c}+L\right]$ will be detected.

\section{Illustrative example}

The model domain has size $L_{x}=500 \mathrm{~m}$ and $L_{y}=400 \mathrm{~m}$ (Fig. 2). The model is discretized with grid cells of $2 \mathrm{~m}$ by $2 \mathrm{~m}$ in both $x$ - and $y$-direction. The hypothetical landfill is located at $30 \leq x \leq 50 \mathrm{~m}$ and $180 \leq y \leq 220 \mathrm{~m}$ in the model domain. The monitoring wells are located in the rectangle $60 \leq x \leq 450 \mathrm{~m}$ and $180 \leq y \leq 220 \mathrm{~m}$. In order to achieve a detailed comparison between analytical and simulation models in terms of estimated concentrations and detection probability values the distance between the monitoring wells is set to $10 \mathrm{~m}$ in the $x$-direction and $2 \mathrm{~m}$ in the $y$-direction.

The boundary conditions of the groundwater flow are: zero flux on top and bottom boundaries, and constant head along left and right boundaries. The head values at $x=0 \mathrm{~m}$ and $x=500 \mathrm{~m}$ were chosen to result in a macroscopically constant hydraulic gradient of 0.001 . The porosity is equal to 0.25 . The average conductivity $K$ is set to $10 \mathrm{~m} /$ day and for homogeneous aquifers, the $y$-location of the leak is the only random input to the model. For the heterogeneous aquifer, uncertainties due to leak location and heterogeneity are considered.

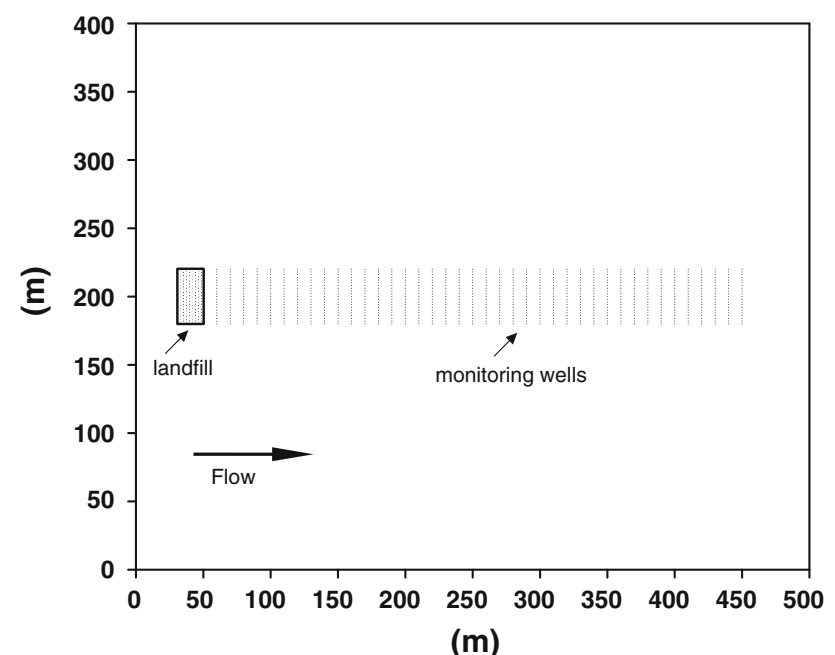

Fig. 2 Flow and transport domain with 840 monitoring wells
Random conductivity fields are generated using the turning bands method (Mantoglou and Wilson 1982). The value of $\mu_{Y}$ is set to 2.3 , whereas the variance of $Y, \sigma_{Y}^{2}$, is assigned four different values, namely $0.2,0.4,1.0$ and 1.5, respectively. The value of $\mu_{Y}=2.3$ corresponds to a geometric mean of the conductivity of $10 \mathrm{~m} /$ day; the isotropic covariance of $Y$ is chosen to be an exponential form with a correlation length $\lambda=15 \mathrm{~m}$.

For the transport model, a condition of a zero dispersive flux is imposed on the top and bottom boundaries, and the initial background concentration in the model domain is set to zero. Since the flow direction is parallel to the $x$ axis, the only source dimension that is treated as a random variable is its $y$ coordinate. Potential leak locations occur along the down gradient edge of the landfill. The leak is assumed to be a point source, as it would result in a plume which is most difficult to detect, and the source location is drawn from a uniform probability distribution between $y$ coordinates of $180 \leq y \leq 220 \mathrm{~m}$ for each Monte Carlo run. Calculations are carried out for two types of leak, namely, instantaneous and continuous leaks. The initial concentration for the instantaneous leak is assumed to be $1 \mathrm{mg} / \mathrm{l}$ whereas for the continuous the leak case injection rate is set to $1 \mathrm{mg} / \mathrm{l} / \mathrm{day}$. The threshold concentration at which detection occurs is set at $0.5 \%$ of the initial source concentration. Contaminants are assumed to be completely mixed over the depth of the aquifer. The ratio between $\alpha_{L}$ and $\alpha_{T}$ is assumed to be 10 (Bear 1972), and $\alpha_{L}$ is set to 0.1 and $0.5 \mathrm{~m}$.

\section{Results and discussion}

Assessment of simulations by analytical methods for the homogeneous case

\section{Instantaneous leak}

For sensitivity analysis, 500, 1,000, 2,000, 4,000, and 8,000 particles are used in order to investigate the appropriate number of particles for estimation of the concentration field. The simulations are performed for the cases where, $\alpha_{L}=0.1 \mathrm{~m}, \alpha_{T}=0.01 \mathrm{~m}$ and $\alpha_{L}=0.5 \mathrm{~m}, \alpha_{T}=0.05 \mathrm{~m}$, respectively. Figure 3 shows the maximum concentration reached by the plume over time for simulations with different numbers of particles on three different levels for $y$, as well as the values that come from the analytical solution for $\alpha_{L}=0.1 \mathrm{~m}, \alpha_{T}=0.01 \mathrm{~m}$. Figure 3 shows simulations with 500, 1,000, 2,000, 4,000 and 8,000 particles. The plume edge (which occurs around $y=204 \mathrm{~m}$ ) is the best using 8,000 particles. Since simulations using 8,000 particles are computationally very expensive, 2,000 particles are used in the rest of the analysis. 
Fig. 3 Maximum

concentrations, over all times, of a plume found by simulation and from the analytical model in the case of an instantaneous leak $(y=200 \mathrm{~m})$ in the homogeneous case for $\alpha_{L}=0.1 \mathrm{~m}, \alpha_{T}=0.01 \mathrm{~m}$ for longitudinal sections along a $y=200 \mathrm{~m}$, b $y=202 \mathrm{~m}$ and $\mathbf{c} y=204 \mathrm{~m}$
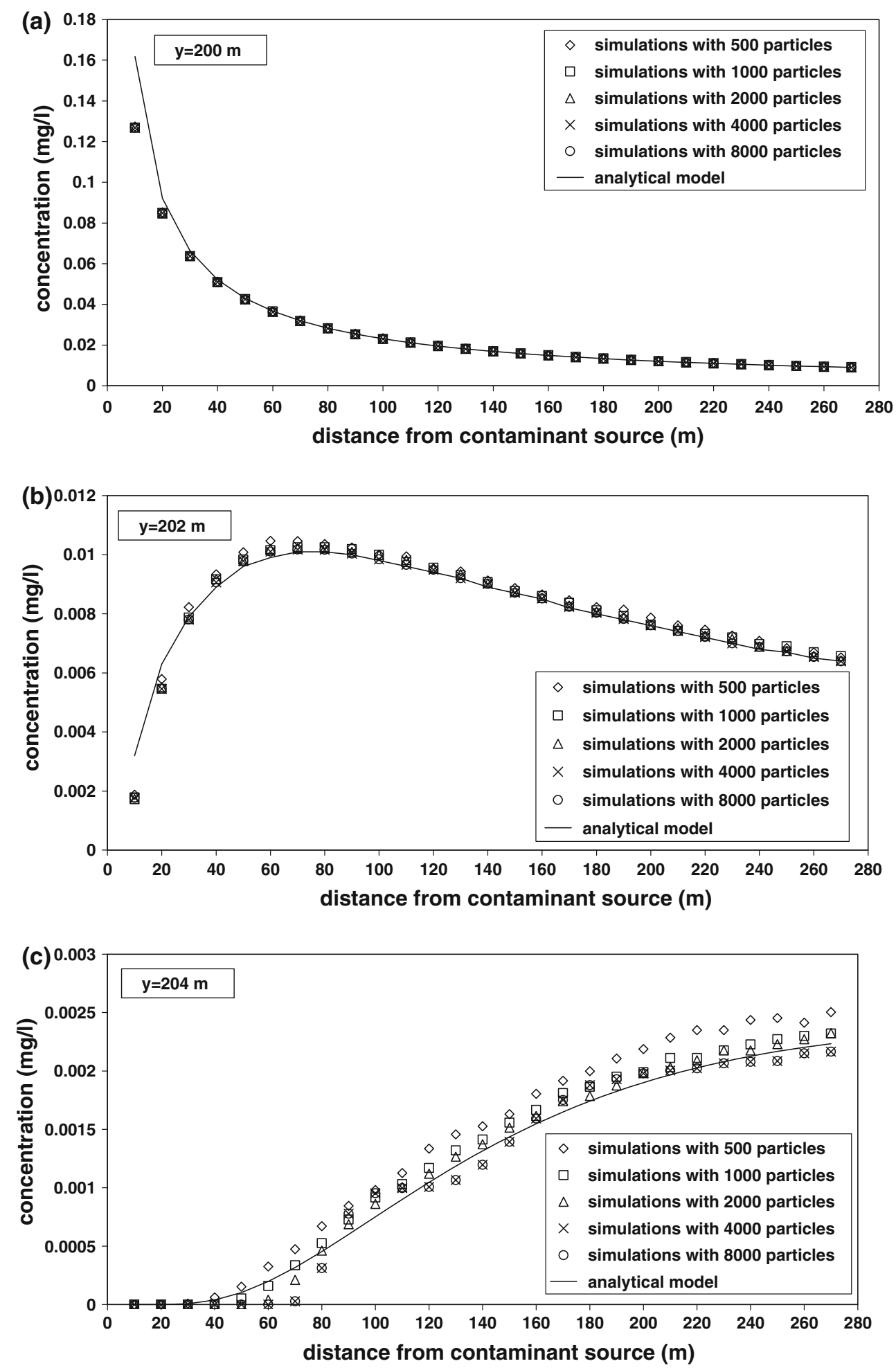

Concentrations obtained by simulation are accurate over most of the plume length. However, near the source there is a slight discrepancy between the simulation and analytical models especially when the dispersivity is low. The plumes are narrow close to the source and widen as they move away. Therefore, close to the source the concentration determined by the analytical model is more peaked. The averaging of the analytical solution using Simpson's rule overestimates the average concentration. This leads to higher discrepancy between the two models in the low 
Fig. 4 Comparison of detection probability at selected wells computed by simulation and analytical models for an instantaneous leak in the homogeneous case a $\alpha_{L}=0.1 \mathrm{~m}, \alpha_{T}=0.01 \mathrm{~m}$ and b $\alpha_{L}=0.5 \mathrm{~m}, \alpha_{T}=0.05 \mathrm{~m}$
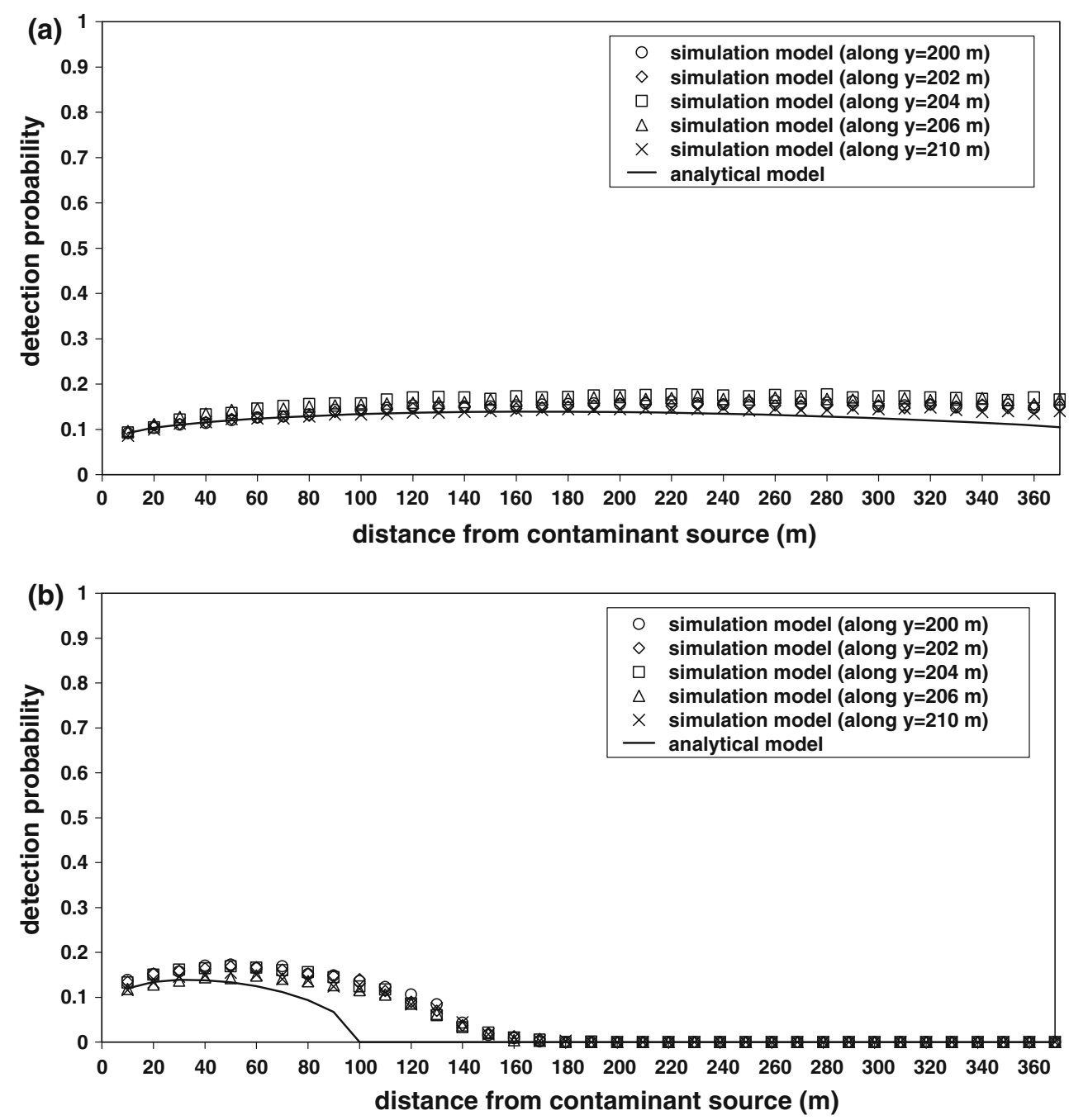

dispersive medium $\left(\alpha_{L}=0.1 \mathrm{~m}, \alpha_{T}=0.01 \mathrm{~m}\right.$, shown in Fig. 3) compared to the highly dispersive medium ( $\alpha_{L}=0.5 \mathrm{~m}, \alpha_{T}=0.05 \mathrm{~m}$, not shown).

Figure 4 shows a comparison of detection probabilities computed by the simulation and the analytical model at the selected wells for both dispersivity cases. The possible leak locations are now located at $x=50 \mathrm{~m}$ and randomly distributed over $180 \leq y \leq 220 \mathrm{~m}$. The values estimated by the simulation model are compatible with those obtained from the analytical model. The slight discrepancy seen in the graphs especially in Fig. $4 \mathrm{~b}$ is due to the fact that the plume edges are not as sharply defined as in the analytical model.

\section{Continuous leak}

Plumes originate from a continuous leak located at $x=50 \mathrm{~m}$ and $y=200 \mathrm{~m}$ with an injection rate of $1 \mathrm{mg} / \mathrm{l} /$ day. In this case the plume converges to a steady state. As in the instantaneous leak case, the simulation model estimated the concentration correctly over most of the plume length (see Fig. 5, which shows the concentration profile on the long run, i.e., close to its steady state). The discrepancy between the simulation and analytical model estimations close to the source, particularly in the low dispersive case, is due to the slender nature of the plume when it is close to the source. The results are representative for the case where $\alpha_{L}=0.5 \mathrm{~m}, \alpha_{T}=0.05 \mathrm{~m}$ as well. Figures 6 and 7 present detection probabilities at selected wells for continuous leaks in the homogeneous case for $\alpha_{L}=0.1 \mathrm{~m}, \alpha_{T}=0.01 \mathrm{~m}$ and $\alpha_{L}=0.5 \mathrm{~m}, \alpha_{T}=0.05 \mathrm{~m}$, respectively. The possible leak locations are at $x=50 \mathrm{~m}$ and $180 \leq y \leq 220 \mathrm{~m}$. The discrepancy between the analytical and simulation model is less than in the instantaneous case. The particle-tracking procedure used for the continuous case is based on an 'addition trick' which goes back to Kinzelbach (1986). Instead of starting new 'independent' plumes at fixed time intervals, the new plumes are identical copies of one plume of say $N=2,000$ particles which is followed at fixed intervals during a certain time span. The domain is divided into a number of cells, and the 
Fig. 5 Concentration profile of a plume at its steady state following from simulation and analytical models from a continuous leak in the homogeneous case with $\alpha_{L}=0.1 \mathrm{~m}, \alpha_{T}=0.01 \mathrm{~m}$ for longitudinal sections along a $y=200 \mathrm{~m}$ and $\mathbf{b} y=206 \mathrm{~m}$
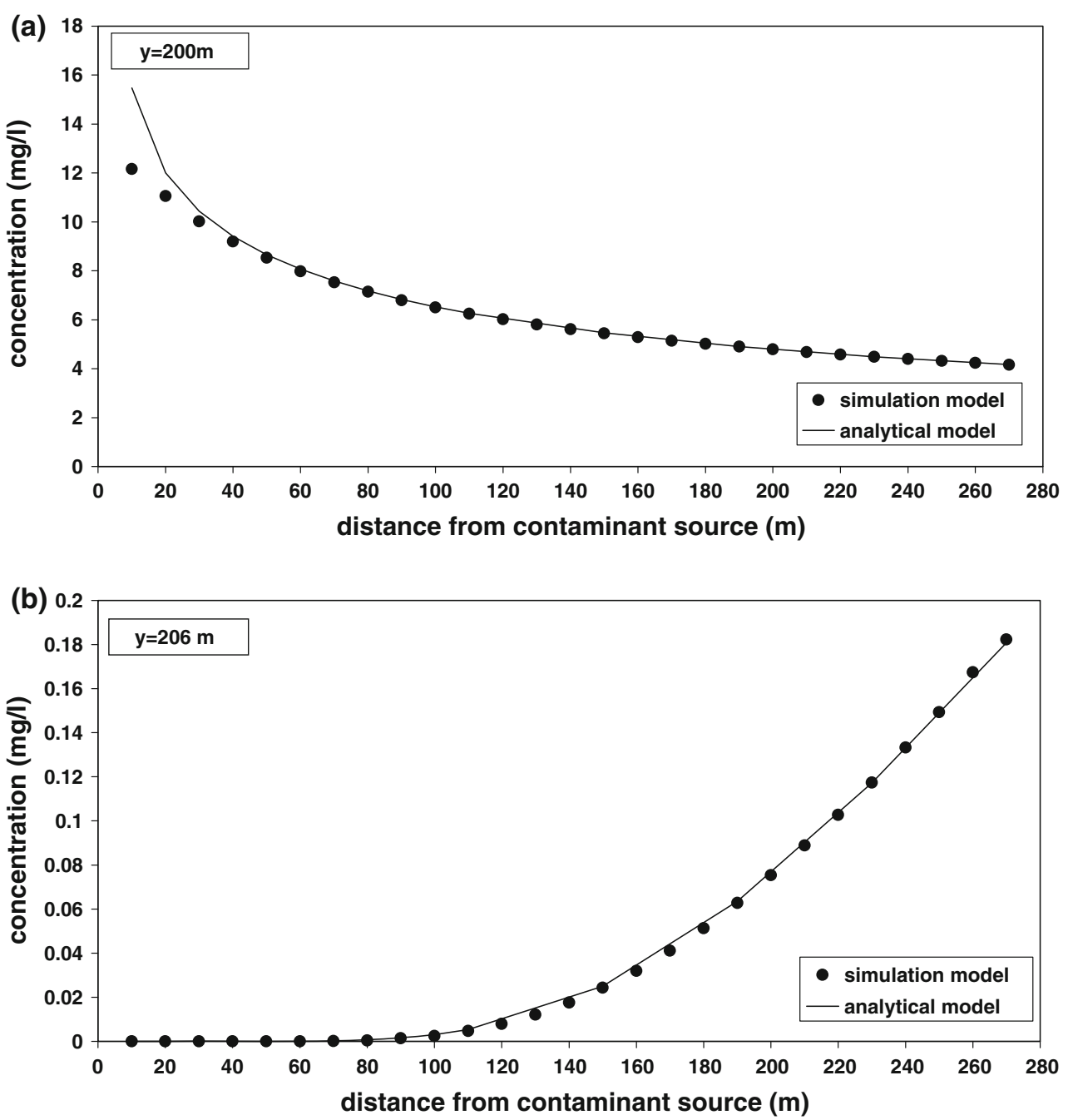

information that is kept along the way are the numbers of particles in each cell (i.e., the concentration at the cells is registered). The updating from one time interval to the next consists of adding the new positions of the foremost plume. In a rather efficient way-after $n$ time intervals only the positions of $N$ particles have to be known, whereas the total number of the whole plume in fact consists of $n N$ particles-this yields better representations of plumes in the continuous case.

Assessment of simulations by analytical methods for the heterogeneous case

The results of the analytical and simulation models described are expressed in terms of concentration profiles along the specified longitudinal sections and plots of the detection probability as a function of the distance from the contaminant source. The goal is to determine: (1) how good is the mean concentration as a predictor of the concentration at a given monitoring well location, and (2) how accurate is it to use the mean concentration in computing the detection probability of a plume by a given well. The computations are carried out for eight scenarios. Table 1 summarizes the parameters for the scenarios considered.

\section{Instantaneous leak}

The concentration field observed in a single heterogeneous aquifer is considered as a realization of a stochastic process, whereas the ensemble mean represents the average behavior of solute plumes in a large number of statistically identical aquifers. The observed concentration distribution does not show a smooth curve as the ensemble concentration. Hence the ensemble mean is not sufficient for the description the concentration field and a successful prediction should be made by computing the uncertainty bounds (i.e., the $95 \%$ confidence intervals). Figure 8 presents the concentration profile at given monitoring wells for three single realizations, the ensemble mean concentration over 700 simulations and their 95\% (empirical) confidence interval along with the mean concentration 
Fig. 6 Comparison of detection probability at selected wells computed by simulation and analytical models for a continuous leak in the homogeneous case $\left(\alpha_{L}=0.1 \mathrm{~m}, \alpha_{T}=0.01 \mathrm{~m}\right)$

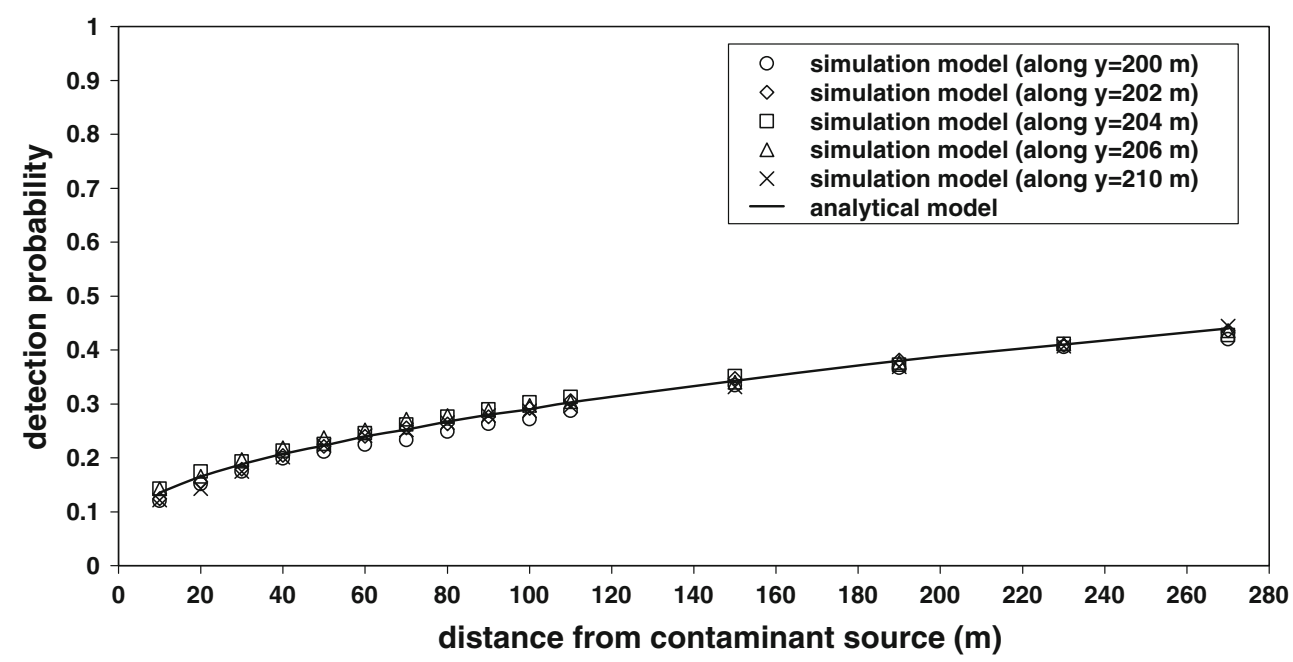

Fig. 7 Comparison of detection probability at selected wells computed by simulation and analytical models for a continuous leak in the homogeneous case $\left(\alpha_{L}=0.5 \mathrm{~m}, \alpha_{T}=0.05 \mathrm{~m}\right)$ a along $y=200 \mathrm{~m}$ and b along $y=210 \mathrm{~m}$
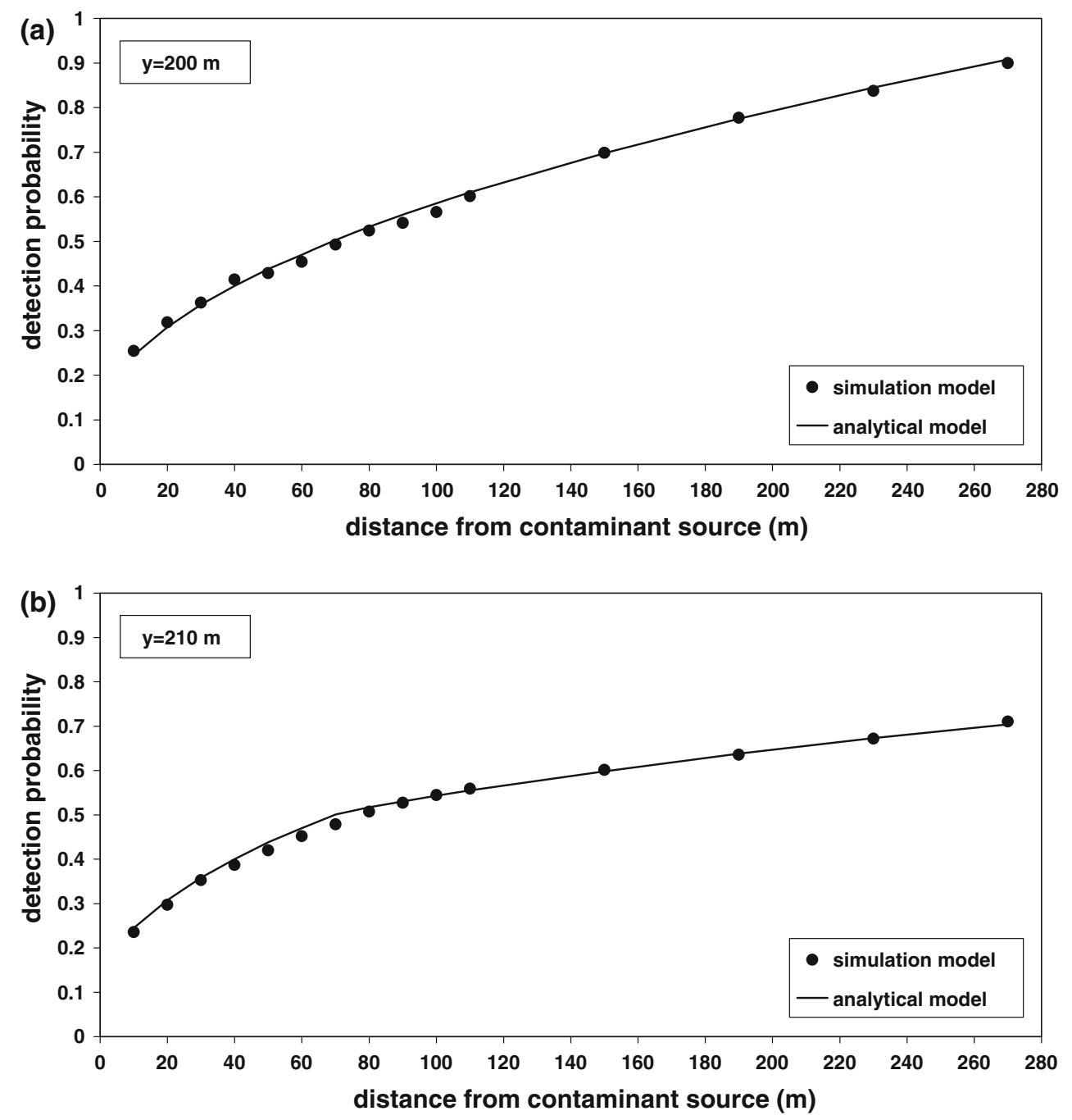

computed by the analytical model for Case 1 a and Case $2 \mathrm{~d}$. Case 1a represents the lowest while Case $2 \mathrm{~d}$ represents the highest dispersive and heterogeneous medium among the scenarios considered.
The average concentrations computed by the two models are close to each other and present smooth curves compared to concentrations of single realizations. The concentration of a single realization is relatively scattered 
Table 1 Parameters used in simulation and analytical models for computations for heterogeneous aquifers

\begin{tabular}{|c|c|c|c|c|c|c|c|c|}
\hline & \multicolumn{4}{|c|}{ Simulation model } & \multicolumn{4}{|c|}{ Analytical model } \\
\hline & $\begin{array}{l}\text { Longitudinal } \\
\text { dispersivity, } \\
\alpha_{L}(\mathrm{~m})\end{array}$ & $\begin{array}{l}\text { Transversal } \\
\text { dispersivity, } \\
\alpha_{T}(\mathrm{~m})\end{array}$ & $\begin{array}{l}\text { Mean } \\
\text { of } Y, \mu_{Y}\end{array}$ & $\begin{array}{l}\text { Variance } \\
\text { of } Y, \sigma_{Y}^{2}\end{array}$ & $\begin{array}{l}\text { Correlation } \\
\text { length, } \lambda(\mathrm{m})\end{array}$ & $\begin{array}{l}\text { Mean } \\
\text { velocity, } \\
v(\mathrm{~m} / \text { day })\end{array}$ & $\begin{array}{l}\text { Longitudinal } \\
\text { macro-dispersivity, } \\
A_{L}(\mathrm{~m})\end{array}$ & $\begin{array}{l}\text { Transversal } \\
\text { macro-dispersivity, } \\
A_{T}(\mathrm{~m})\end{array}$ \\
\hline Case1a & 0.1 & 0.01 & 2.3 & 0.2 & 15 & 0.04 & 3.1 & 0.013 \\
\hline Case1b & 0.1 & 0.01 & 2.3 & 0.4 & 15 & 0.04 & 6.1 & 0.016 \\
\hline Case1c & 0.1 & 0.01 & 2.3 & 1.0 & 15 & 0.04 & 15.1 & 0.026 \\
\hline Case1d & 0.1 & 0.01 & 2.3 & 1.5 & 15 & 0.04 & 22.6 & 0.034 \\
\hline Case2a & 0.5 & 0.05 & 2.3 & 0.2 & 15 & 0.04 & 3.5 & 0.066 \\
\hline Case2b & 0.5 & 0.05 & 2.3 & 0.4 & 15 & 0.04 & 6.5 & 0.082 \\
\hline Case2c & 0.5 & 0.05 & 2.3 & 1.0 & 15 & 0.04 & 15.5 & 0.131 \\
\hline Case2d & 0.5 & 0.05 & 2.3 & 1.5 & 15 & 0.04 & 23 & 0.171 \\
\hline
\end{tabular}
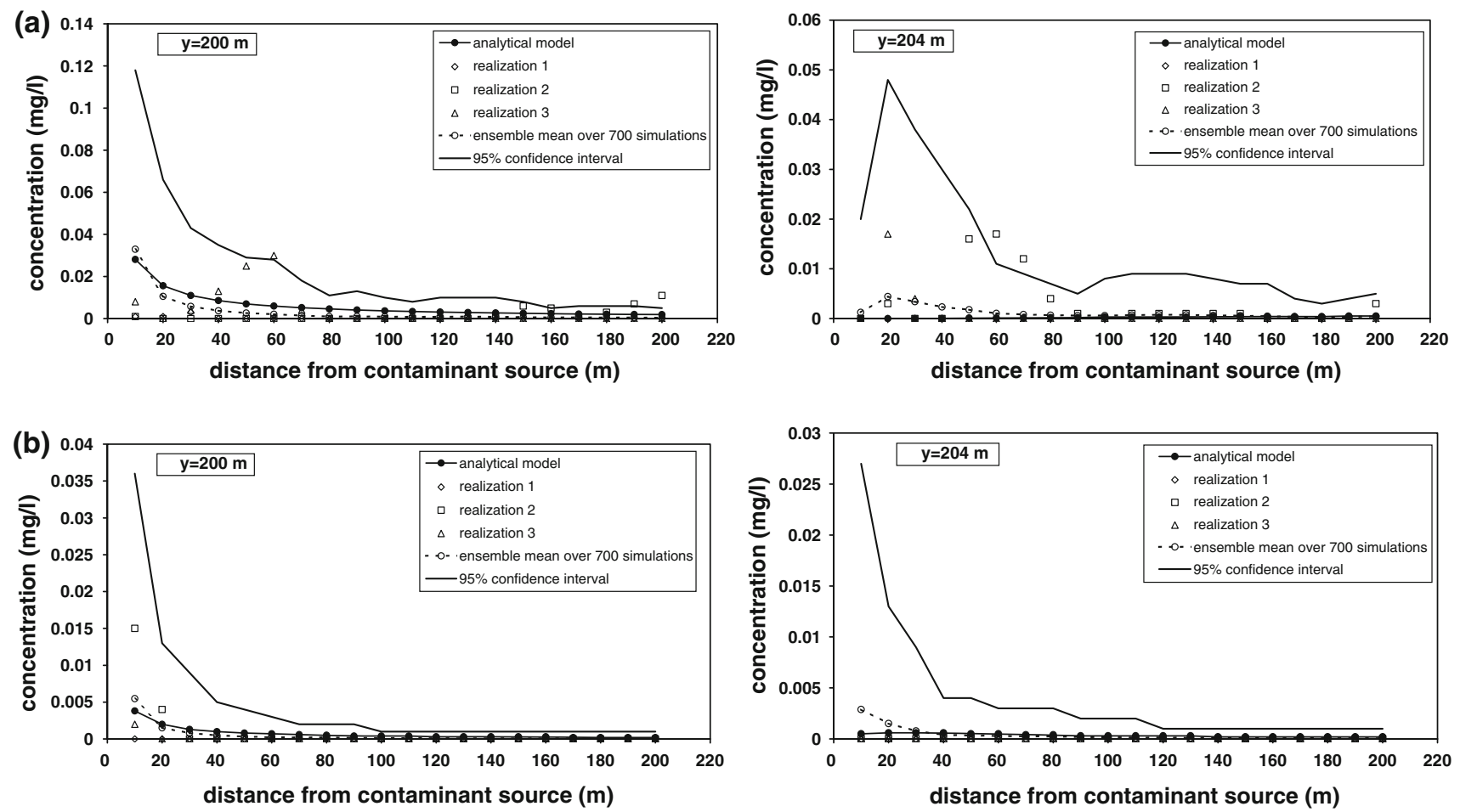

Fig. 8 Concentration profile from simulation and analytical models of an instantaneous leak $(y=200 \mathrm{~m})$ in the heterogeneous case for longitudinal sections along $y=200 \mathrm{~m}$ (left column) and $y=204 \mathrm{~m}$ (right column) a Case 1a b Case $2 \mathrm{~d}$

as expected, since each realization has a different plume velocity and a different spreading. The $95 \%$ confidence interval is wider close to the source: in all cases uncertainty in concentration prediction decreases with distance from the source. The ensemble standard deviation in the concentration is higher near the source and reduces significantly as plume moves further away. Near the source the plume is narrow and has a large degree of freedom to spread in different forms from one realization to another. However, further away from the source the plume widens and since it covers a larger area the degree of freedom to spread is not that high and the uncertainty is less. The $95 \%$ confidence interval is narrower towards the edge of the plume $(y=204 \mathrm{~m})$ for the same reason. The discrepancy between the two models is more pronounced in the low dispersive medium.

Figure 9 shows a comparison of detection probabilities for four of the eight cases - the cases not shown are similar to Case 1a respectively Case 2a. A discrepancy occurs between the analytical and simulation models. The analytical model uses macro-dispersivities to compute the mean concentration, which is smoother and produces relatively 

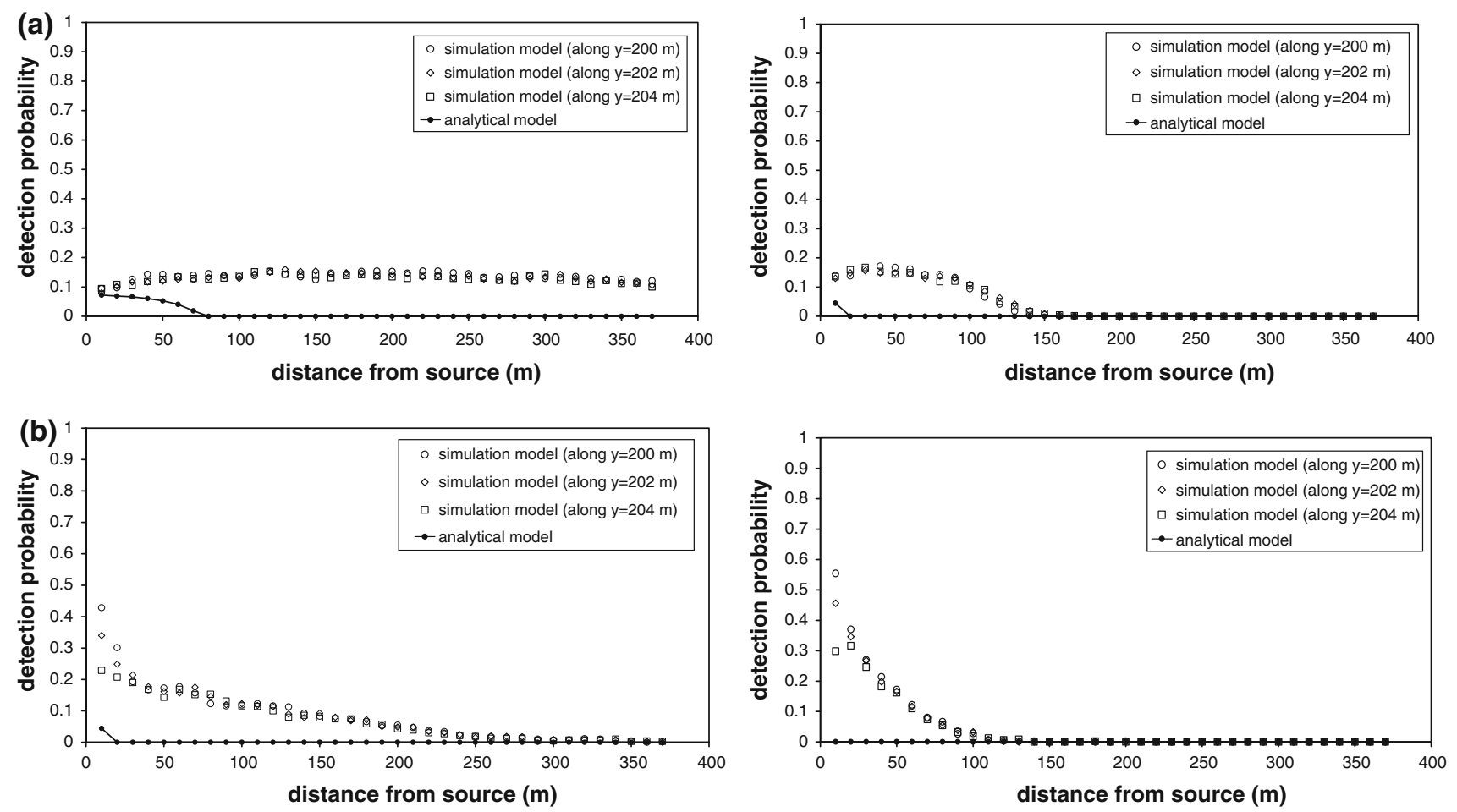

Fig. 9 Comparison of detection probability at selected well computed by simulation and analytical models for an instantaneous leak in the heterogeneous case a Case 1a and Case 2a, b Case 1d and Case 2d

wider plumes. This results in a lower detection probability than those obtained by the simulation model. Homogenization underestimates the plume size and its influence is more pronounced when the dispersivity and/or $\sigma_{Y}^{2}$ increase.

\section{Continuous leak}

This type of leak is mostly considered in monitoring system design at landfill sites unless there are specific data for the type of the leak. Figure 10 presents the comparison of concentration profiles computed by the two models in the case of a continuous leak with an injection rate of $1 \mathrm{mg} / \mathrm{l} /$ day for Case 1a and Case 2d. We only show those, as 1a and $2 \mathrm{~d}$ can be considered as bounds for the remaining cases.

The discrepancy between the average concentration computed by the two models decreases as the dispersivity of the medium increases since the plume gets wider and the concentration gradient is smaller for larger dispersivity. As described above for the instantaneous leak case the 95\% confidence interval is wider close to the source and narrower towards the edge of the plume $(y=208 \mathrm{~m})$ in the continuous leak case as well, since the concentration gradient decreases as the distance from the source increases. However, in this case the influence of heterogeneity is more visible compared to the instantaneous leak case: the confidence interval close to the source appears to be wider when $\sigma_{Y}^{2}$ increases. This is because in the instantaneous leak case the plumes spread faster when the heterogeneity and dispersivity of the medium increases and accordingly the concentration and hence the concentration gradient become smaller.

However, in the case of a continuous leak the continuous injection of contaminants results in higher concentration levels and therefore a larger concentration gradient, which actually reflects the apparent influence of heterogeneity: the uncertainty in concentration prediction increases as the degree of heterogeneity increases. This also explains why the discrepancy between average concentrations computed by the two models is higher than in the instantaneous case.

The detection probability is presented in Figs. 11 and 12. There is a big discrepancy between the detection probabilities computed by the two models. The reason is the overestimation of concentration computed by the analytical model. Therefore the detection probability increases as the heterogeneity increases.

\section{Conclusions}

Simulation and analytical models are used to compute concentration distributions and the associated detection probabilities at given monitoring wells. 

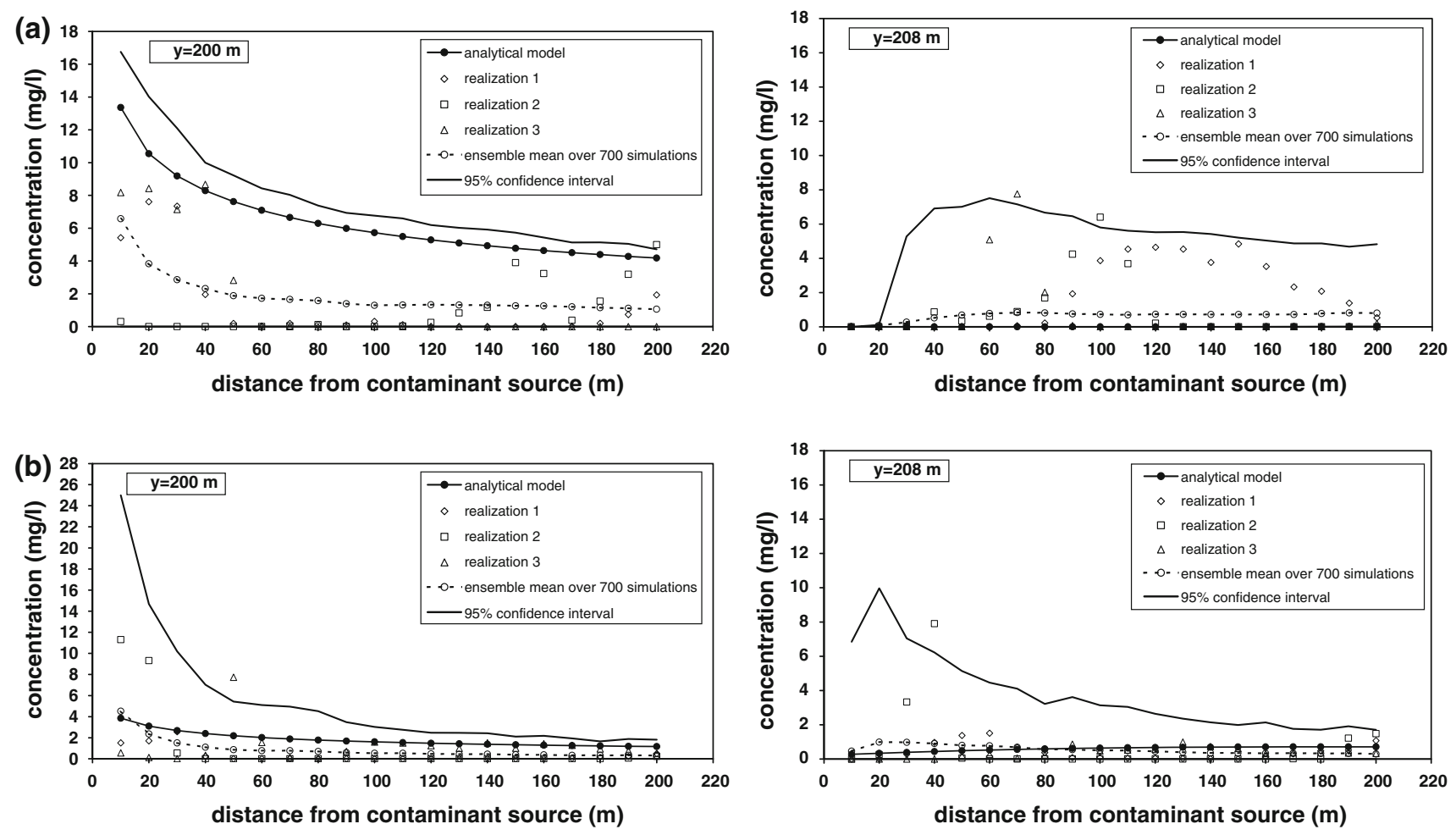

Fig. 10 Concentration profile from simulation and analytical models from a continuous leak $(y=200 \mathrm{~m})$ in the heterogeneous case for longitudinal sections along $y=200 \mathrm{~m}$ (left column) and $y=204 \mathrm{~m}$ (right column) a Case 1a, and b Case $2 \mathrm{~d}$
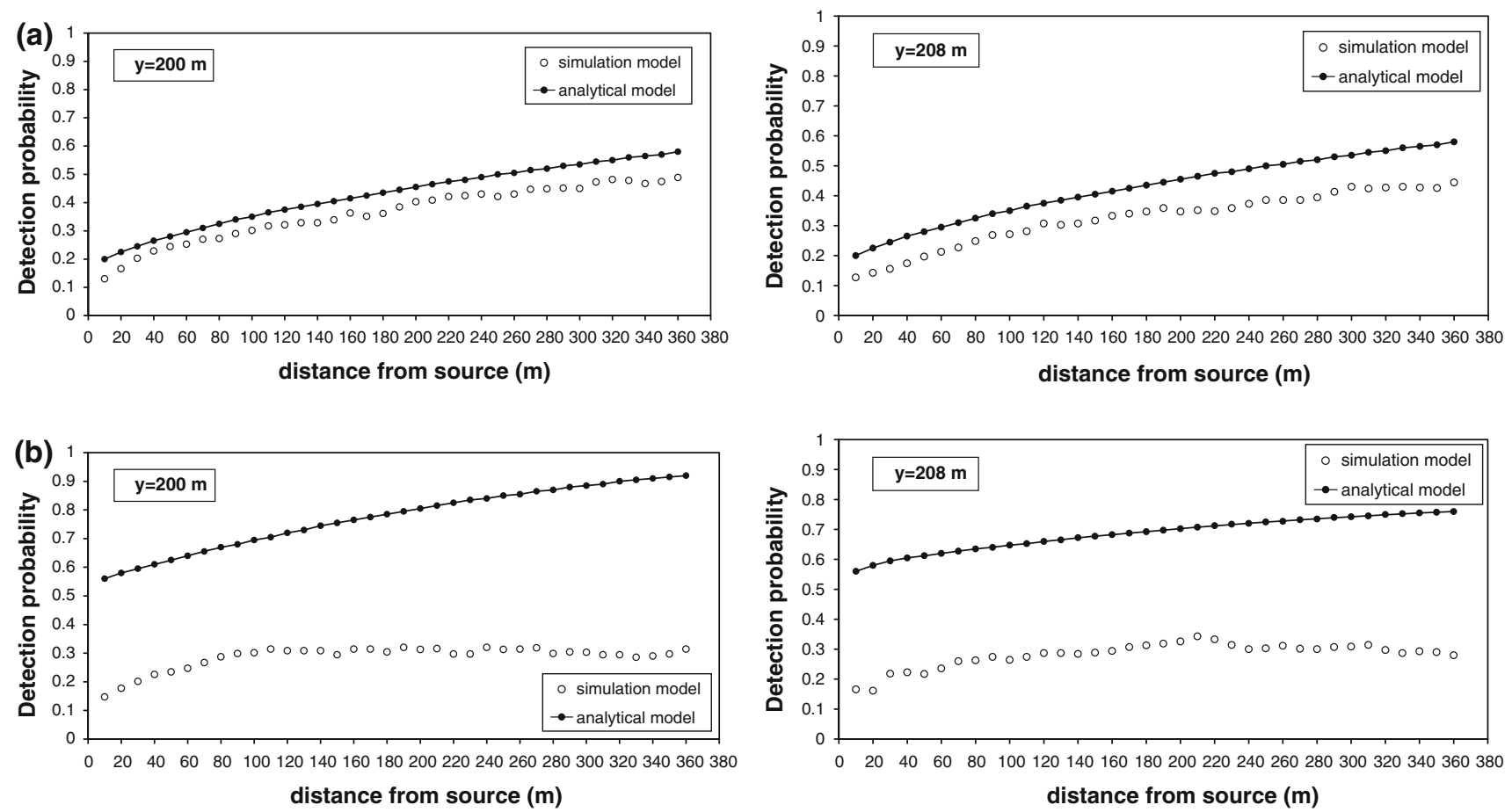

Fig. 11 Comparison of detection probability at selected wells computed by simulation and analytical models for continuous leak in a heterogeneous medium along $y=200 \mathrm{~m}$ (left column) and $y=208 \mathrm{~m}$ (right column) a Case 1a, and b Case $1 \mathrm{~d}$ 

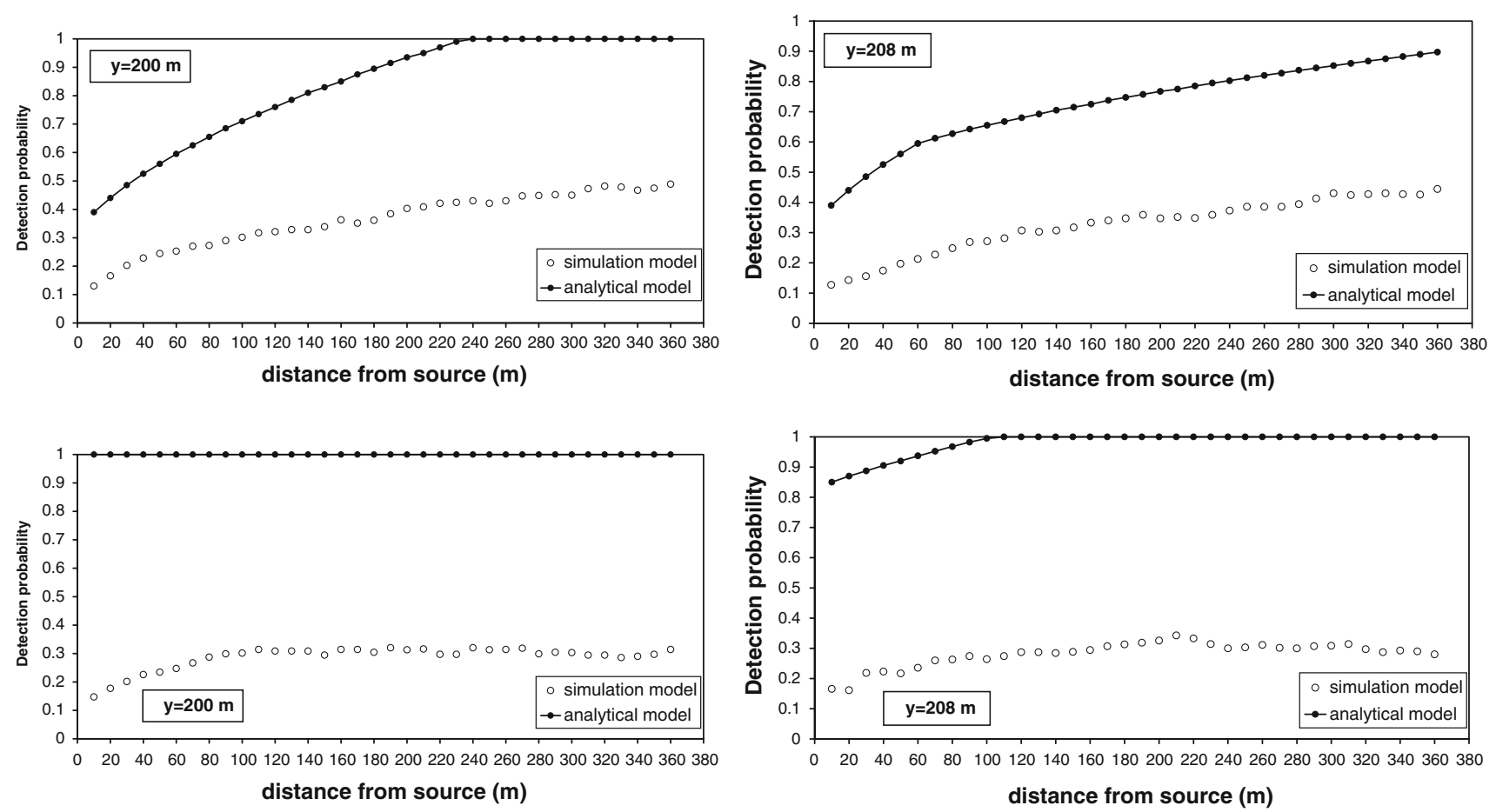

Fig. 12 Comparison of detection probability at selected wells computed by simulation and analytical models for continuous leak in a heterogeneous medium along $y=200 \mathrm{~m}$ (left column) and $y=208 \mathrm{~m}$ (right column) a Case $2 \mathrm{a}$, and b Case $2 \mathrm{~d}$

For homogeneous aquifer conditions, the results show that the simulation model estimates the concentration correctly over the plume length, except near the source. We have determined the detection probability analytically by introducing the notions of detection regions and leak regions. Because of the sensitivity of the detection probability quantity, a large number of particles is necessary in the simulation model. The results in terms of detection probabilities match quite well, in particular for the continuous leak.

For a heterogeneous aquifer, the use of macro-dispersion coefficients in an analytical model to describe the concentration distribution leads to a discrepancy of both the analytical and the simulation model, in particular in the continuous leak case. The mean concentration plume that results from such an approximation is smooth due to homogenization. This overlooks the fluctuations in the concentration field and is consequently reflected in the detection probability. Modeling contaminant transport using macro-dispersivities can describe the average concentration distribution fairly good under small degree of variability; however, this approach is insufficient in monitoring system design when the degree of variability is large. The discrepancy between the detection probabilities by the two models is significant, particularly when the dispersivity and heterogeneity of the medium is large. Therefore, despite the computational expenses, the simulation model is more appropriate for monitoring system design under conditions of heterogeneity.
Acknowledgments The financial support by DIOC Water of the Delft University of Technology and the company Tauw for this research is gratefully acknowledged.

Open Access This article is distributed under the terms of the Creative Commons Attribution Noncommercial License which permits any noncommercial use, distribution, and reproduction in any medium, provided the original author(s) and source are credited.

\section{Appendix A: Determining the plume width at fixed well distance}

The (vertical) width of the plume at time $t$ at a well distance $x_{\mathrm{mw}}$ can be found by solving $C\left(x_{\mathrm{mw}}, y, t\right)=C_{\mathrm{TH}}$ for $y$ which gives,

$y^{2}=4 \alpha_{T} v_{x} t\left[\ln \left(\frac{C_{0} 1}{C_{\mathrm{TH}} 4 \pi \sqrt{\alpha_{L} \alpha_{T}} v_{x} t}\right)-\frac{\left(x_{\mathrm{mw}}-v_{x} t\right)^{2}}{4 \alpha_{L} v_{x} t}\right]$.

Define the abbreviation

$A:=\frac{C_{0}}{C_{\mathrm{TH}} 4 \pi \sqrt{\alpha_{L} \alpha_{T}}}$.

This gives

$y=g(t)=\sqrt{4 \alpha_{T} v_{x} t\left(\ln A-\ln v_{x} t\right)-\frac{\alpha_{T}}{\alpha_{L}}\left(x_{\mathrm{mw}}-v_{x} t\right)^{2}}$. 
To find the maximum $l\left(l=g\left(t_{\max }\right)\right)$, differentiate $g$ with respect to $t$ : one has to solve $g^{\prime}(t)=0$. This is not analytically feasible. Note that, for fixed $t$, the contours $C(x, y$, $t)=$ constant are ellipses. One would expect the plume has its maximal width at distance $x_{\mathrm{mw}}$ when the center of this ellipse is at $x_{\mathrm{mw}}$, which happens at $t=x_{\mathrm{mw}} / v_{x}$. Using numerical approximations it is found that the width of the plume for this $t$ is very close to the optimal width. This is the way the maximal width $2 l$ of the plume is calculated in the analytical model.

\section{Appendix B: Corrections for boundary effects}

Here we calculate the corrections to Eq. 8. We refer to Fig. 1 to see what is going on. In all cases $P_{d(\mathrm{mw})}$ is simply the portion of the interval $\left[y_{c}-L, y_{c}+L\right]$ from which leaks will be detected at well location $\left(x_{\mathrm{mw}}, y_{\mathrm{mw}}\right)$. In the middle of the interval, this is just $2 l / 2 L$, where $2 l$ is the width of the plume at distance $x_{\mathrm{mw}}$, but at the boundary $\left(y_{\mathrm{mw}} \approx y_{c} \pm L\right)$ this probability decreases. If $l \leq L$ and, say $y_{\mathrm{mw}}+l \geq y_{c}+$ $L$, the leaks in $\left[y_{c}+L, y_{\mathrm{mw}}+l\right]$, which is an interval of length $\left(y_{\mathrm{mw}}+l-y_{c}-L\right)$ should not be counted and,

$P_{d(\mathrm{mw})}=\frac{2 l-\left(\left(y_{\mathrm{mw}}+l\right)-\left(y_{c}+L\right)\right)}{2 L}=\frac{l+L-y_{\mathrm{mw}}+y_{c}}{2 L}$

Likewise if $y_{\mathrm{mw}}-l \leq y_{c}-L$ the detection probability equals:

$P_{d(\mathrm{mw})}=\frac{2 l-\left(\left(y_{c}-L\right)-\left(y_{\mathrm{mw}}-l\right)\right)}{2 L}=\frac{l+L-y_{c}+y_{\mathrm{mw}}}{2 L}$

If $L \leq l \leq 2 L$ and, if $L-l \leq y_{\mathrm{mw}}-y_{c} \leq l-L$ the detection probability $P_{d(\mathrm{mw})}$ is 1 .

The boundary cases can be handled as above.

\section{References}

Bagchi A (1994) Design, construction, and monitoring of landfills. Wiley, Canada

Bear J (1972) Dynamics of fluid in porous media. Elsevier, New York

Chofqi A, Younsi A, El Kbir L, Mania J, Mudry J, Veron A (2004) Environmental Impact of an urban landfill on a coastal aquifer (El Jadida, Morocco). J Afr Earth Sci 39:509-516

Dagan G (1984) Solute transport in heterogeneous porous media. J Fluid Mech 145:813-833

Dagan G (1986) Statistical theory of groundwater flow and transport: pore to laboratory, laboratory to formation, and formation to regional scale. Water Resour Res 22:120S-134S

Elfeki AMM (1996) Stochastic characterization of geological heterogeneity and its impact on groundwater contaminant transport. Balkema Publishers, Rotterdam
Gelhar LW, Axness CL (1983) Three-dimensional stochastic analysis of macro-dispersion. Water Resour Res 19:161-180

Gelhar LW, Gutjhar AL, Naff RL (1979) Stochastic analysis of macrodispersion in a stratified aquifer. Water Resour Res 15:1387-1397

Haug A, Petrini RH, Grisak GE, Klahsen K (1989) Application of geostatistical methods to assess position and spacing of groundwater monitoring wells. Proc., petroleum hydrocarbons and organic chemicals in groundwater: prevention, detection and restoration, National Water Well Association, pp 535-548

Hu BX, Huang H, Zhang D (2002) Stochastic analysis of solute transport in heterogeneous, dual-permeability media. Water Resour Res 38(9):1175

Hudak PF (2001) Effective contaminant detection networks in uncertain groundwater flow fields. Waste Manag 21:309-312

Hudak PF (2002) Efficiency comparison of graphical approaches for designing contaminant detection networks in groundwater. Water Resour Res 38(12):1282

Hudak PF, Loaiciga HA (1993) A location modelling approach for groundwater monitoring network augmentation. Water Resour Res 28:643-649

Kapoor V, Gelhar LW (1994a) Transport in three-dimensionally heterogeneous aquifers 1 . Dynamics of concentration fluctuations. Water Resour Res 30:1775-1788

Kapoor V, Gelhar LW (1994b) Transport in three-dimensionally heterogeneous aquifers 2. Predictions and observations of concentration fluctuations. Water Resour Res 30:1789-1801

Kinzelbach W (1986) Groundwater modelling. Development in water Science 25. Elsevier, Amsterdam

Mantoglou A, Wilson JL (1982) The turning bands method for simulation of random fields using line generation by spectral method. Water Resour Res 18:1379-1394

Massmann J, Freeze RA (1987) Groundwater contamination from waste management sites: the interaction between risk-based engineering design and regulatory policy, 1, Methodology. Water Resour Res 23:351-367

McLaughlin D, Ruan F (2001) Macrodispersivity and large-scale hydrogeologic variability. Transp Porous Med 42:133-154

Meyer PD, Valocchi AJ, Eheart JW (1994) Monitoring network design to provide initial detection of groundwater contamination. Water Resour Res 30:2647-2659

Mikac N, Cosovic B, Ahel SA, Toncic Z (1998) Assessment of groundwater contamination in the vicinity of a municipal waste landfill (Zagreb, Croatia). Water Sci Technol 37:37-44

Rouhani S, Hall TJ (1988) Geostatistical schemes for groundwater sampling. J Hydrol 103:85-102

Rubin Y (1990) Stochastic modelling of macrodispersion in heterogeneous porous media. Water Resour Res 26:133-141

Storck P, Eheart JW, Valocchi AJ (1997) A method for optimal location of monitoring wells for detection of groundwater contamination in three-dimensional heterogeneous aquifers. Water Resour Res 33:2081-2088

Tatsi AA, Zouboulis AI (2002) A field investigation of the quantity and quality of leachate from a municipal solid waste landfill in a Mediterranean climate (Thessaloniki, Greece). Adv Environ Res 6:207-219

Thompson AFB, Gelhar LW (1990) Numerical simulation of solute transport in three-dimensional, randomly heterogeneous porous media. Water Resour Res 26:2451-2562

Vomvoris EG, Gelhar LW (1990) Stochastic analysis of the concentration variability in a three-dimensional heterogeneous aquifer. Water Resour Res 26:2591-2602 